\title{
Reversing Type I Migration in Gap Shadows
}

\author{
Hannah Jang-Condell \\ Dept. of Physics \& Astronomy, University of Wyoming \\ 1000 E University, Dept 3905 \\ Laramie, WY 82071, USA \\ email: hjangcon@uwyo.edu
}

\begin{abstract}
Type I migration refers to the radial drift of a sub-Jupiter mass planet resulting from tidal interactions with a protoplanetary disk. It results in the rapid inward migration of small planets and planet cores through the disk. Type I migration is so rapid compared to disk dissipation time scales that explaining distant planets, such as the HR 8799 planets and Jupiter itself, is problematic because their growing cores should have been lost. Here, we present a scenario for solving the Type I migration problem. As a planet grows in mass, its Type I migration rate should increase, assuming that disk properties are not significantly altered by the forming planet. But a growing planet clears some, but not all, material from its orbital path, creating a partial gap in the disk. The trough of a partially cleared gap such as this is shadowed from stellar illumination while the far side of the gap is illuminated. Since stellar irradiation is the primary heat source of passively accreting protoplanetary disks, gap self-shadowing can significantly change the local temperature profile. This change to the local temperature gradient can significantly slow, or even reverse Type I migration.
\end{abstract}

Keywords. planetary systems: formation, Type I migration, planetary dynamics

Type I migration occurs because of exchange of angular momentum between a planet and a viscous gas disk. The planet loses angular momentum to the outer disk and gains angular momentum from the inner disk, with the direction of migration dependent on the balance of the torques. Ward (1997) showed that the migration is inward in typical protoplanetary disks because the pressure support shifts the position of the Lindblad resonances systematically inward. So long as the pressure gradient is negative (pressure decreasing with radius) inward migration results. Here, we show that shadowing in gaps can sufficiently reverse the temperature gradient so as to reverse the balance of torques.

Jang-Condell \& Sasselov (2005) showed that shadowing near local planet perturbations in disks change the temperature gradient to slow Type I migration near planets by up to a factor of 2 , but did not include the effects of gap clearing by the planet. Planets of about a Jupiter mass or larger can halt Type I migration by completely clearing their resonances of disk material. Then, the planet undergoes Type II migration, moving in lock step with the accretion of the disk onto the star. At intermediate masses $\left(\gtrsim 30 M_{\oplus}\right)$, the planet does not fully clear a gap, but clears a partial gap, where the density is nonzero but less than what it would be in an unperturbed disk. Shadowing and heating on this gap is more substantial than that produced by the local perturbations modeled in Jang-Condell \& Sasselov (2005), and Type I migration rates should be more affected.

In Jang-Condell \& Turner (2012), we modeled the tempertures in gaps produced by planets, accounting for shadowing and illumination effects and self-consistently calculating the density and temperature structure of the gap. The resulting temperatures for varying planet mass and position are shown in Figure 1. As the depth of the gap increases, so does the magnitude of the midplane temperature perturbation. At $1 \mathrm{AU}$, the temperature decreases in the shadow caused by the gap. At farther distances, the 


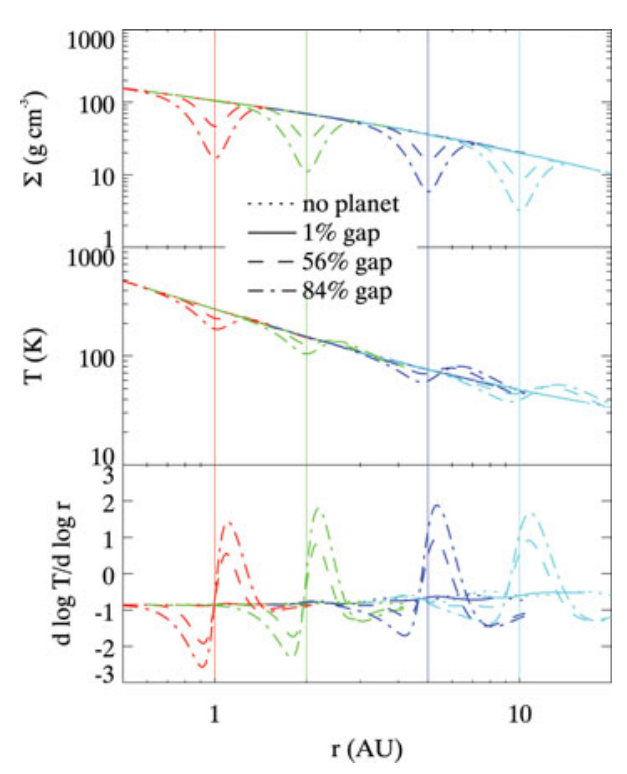

Figure 1. Surface density and midplane temperatures in the vicinity of partial gaps produced by planets at 1 (red), 2 (green), 5 (blue), and 10 (cyan) AU. The vertical lines show the position of the planet.

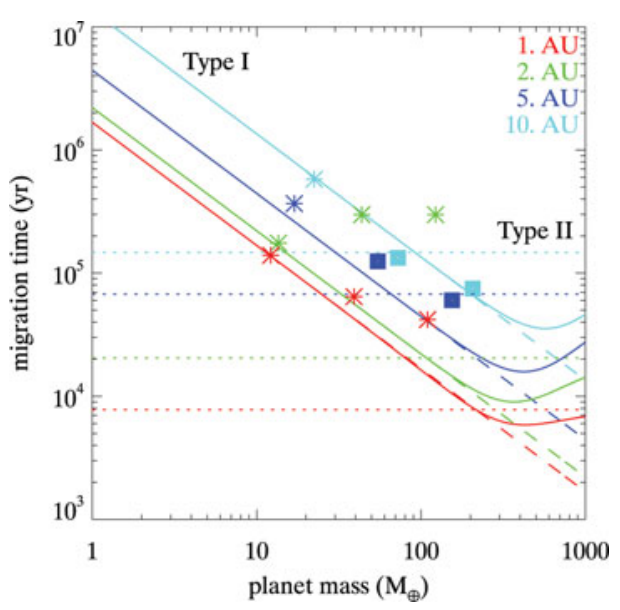

Figure 2. Type I migration time scales calculated for planets at 1 (red), 2 (green), 5 (blue), and 10 (cyan) AU. Type I and Type II migration rates in the absences of gap shadowing are shown by dashed and dotted lines, respectively, and the solid line shows the transition between the two. The points indicate migration rates calculated accounting for temperature perturbations caused by shadowing in the gaps, with asterisks indicating inward migration and squares indicating outward migration.

temperature decrease at or interior to the planet's distance is paired with a temperature increase outside the planet's orbit. The temperature increase is caused by the illumination of the exposed shoulder of the far side of the gap. The effect is smaller at smaller distances from the star because viscous heating sets a lower bound for the temperature, leading to a smaller shadow to begin with. The paired cooling and heating leads to a local inversion to the temperature profile, as shown in the plot of $d \log T / d \log r$ in the bottom panel of Figure 1. As the temperature gradient becomes increasingly positive, the more the balance of torques will lead to outward rather than inward migration.

In Figure 2, we show the final migration time scales calculated with the inclusion of cooling in the shadows of the gaps carved by planets. Asterisks indicate inward migration while squares indicate outward migration. At $1 \mathrm{AU}$ (red), as the gap size increases, the migration rate increases (time scale decreases) with planet mass, but not as rapidly as compared to the predictions of Type I migration. At 2 AU (green), the migration rate actually decreases with increasing planet mass. At 5 and $10 \mathrm{AU}$, the temeprature gradient is sufficiently reversed to lead to outward migration on rapid time scales.

In conclusion, we have found a way to halt or even reverse Type I migration as planets grow in mass. The timing of the growth of planets may determine which way they migrate. Planets that grow quickly can reverse migration early on and end up very far from the star, whereas planets that grow slowly will migrate inward.

\section{References}

Jang-Condell, H. \& Sasselov, D. D. 2005, ApJ, 619, 1123

Jang-Condell, H. \& Turner, N. J. 2012, ApJ, 749, 153

Ward, W. R. 1997, Icarus, 126, 261 